\title{
NATbox: a network analysis toolbox in $\mathbf{R}$ Shweta S Chavan ${ }^{1}$, Michael A Bauer ${ }^{1}$, Marco Scutari ${ }^{2}$ and Radhakrishnan Nagarajan*3
}

\author{
Address: ${ }^{1}$ UALR/UAMS Joint Bioinformatics Program, University of Arkansas at Little Rock, Arkansas, USA, ${ }^{2}$ Department of Statistical Sciences, \\ University of Padova, Italy and ${ }^{3}$ Department of Biostatistics, University of Arkansas for Medical Science, USA \\ E-mail: Shweta S Chavan - schavan@uams.edu; Michael A Bauer - mabauer@ualr.edu; Marco Scutari - marco.scutari@stat.unipd.it; \\ Radhakrishnan Nagarajan* - rnagarajan@uams.edu \\ ${ }^{*}$ Corresponding author
}

from Sixth Annual MCBIOS Conference. Transformational Bioinformatics: Delivering Value from Genomes

Starkville, MS, USA 20-2I February 2009

Published: 08 October 2009

BMC Bioinformatics 2009, I0(SuppI II):SI4 doi: 10.1I86/I47I-2105-I0-SII-SI4

This article is available from: http://www.biomedcentral.com/I47I-2/05/I0/SII/SI4

(C) 2009 Chavan et al; licensee BioMed Central Ltd.

This is an open access article distributed under the terms of the Creative Commons Attribution License (http://creativecommons.org/licenses/by/2.0), which permits unrestricted use, distribution, and reproduction in any medium, provided the original work is properly cited.

\begin{abstract}
Background: There has been recent interest in capturing the functional relationships (FRs) from high-throughput assays using suitable computational techniques. FRs elucidate the working of genes in concert as a system as opposed to independent entities hence may provide preliminary insights into biological pathways and signalling mechanisms. Bayesian structure learning (BSL) techniques and its extensions have been used successfully for modelling FRs from expression profiles. Such techniques are especially useful in discovering undocumented FRs, investigating non-canonical signalling mechanisms and cross-talk between pathways. The objective of the present study is to develop a graphical user interface (GUI), NATbox: Network Analysis Toolbox in the language R that houses a battery of BSL algorithms in conjunction with suitable statistical tools for modelling FRs in the form of acyclic networks from gene expression profiles and their subsequent analysis.
\end{abstract}

Results: NATbox is a menu-driven open-source GUI implemented in the R statistical language for modelling and analysis of FRs from gene expression profiles. It provides options to (i) impute missing observations in the given data (ii) model FRs and network structure from gene expression profiles using a battery of BSL algorithms and identify robust dependencies using a bootstrap procedure, (iii) present the FRs in the form of acyclic graphs for visualization and investigate its topological properties using network analysis metrics, (iv) retrieve FRs of interest from published literature. Subsequently, use these FRs as structural priors in BSL (v) enhance scalability of BSL across high-dimensional data by parallelizing the bootstrap routines.

Conclusion: NATbox provides a menu-driven GUl for modelling and analysis of FRs from gene expression profiles. By incorporating readily available functions from existing R-packages, it minimizes redundancy and improves reproducibility, transparency and sustainability, characteristic of open-source environments. NATbox is especially suited for interdisciplinary researchers and biologists with minimal programming experience and would like to use systems biology approaches without delving into the algorithmic aspects. The GUI provides appropriate parameter 
recommendations for the various menu options including default parameter choices for the user. NATbox can also prove to be a useful demonstration and teaching tool in graduate and undergraduate course in systems biology. It has been tested successfully under Windows and Linux operating systems. The source code along with installation instructions and accompanying tutorial can be found at http://bioinformatics.ualr.edu/natboxWiki/index.php/Main_Page.

\section{Background}

Classical biological experiments have focused on understanding changes in the expression of single genes across distinct biological states. Such differential gene expression analyses while useful may not provide sufficient insight into their interactions or functional relationships (FRs). Understanding FRs is crucial as genes work in concert as a system as opposed to independent entities. On a related note, phenotype formation is mediated by pathways comprising of complex interactions between several genes as opposed to a single gene. Recent development of high-throughput assays in conjunction with sophisticated computational tools has enabled modelling such interactions and gain system-level understanding.

Several commercial and non-commercial software packages have been developed in the past for modelling gene interactions. Ontology-based packages $[1,2]$ that rely on prior knowledge have been used traditionally to identify pathways enriched in a given experiment from existing documented pathways. Commercial packages (Ingenuity Pathway Analysis, Ingenuity Systems, Redwood City, CA) and (Pathway Studio, Ariadne Genomics, Rockville, MD) provide menu-driven GUI for retrieving functional relationships on a given set of genes from published literature. It is important to note that such techniques draw conclusions based on documented pathways and pooling knowledge across disparate sources. This in turn can render the conclusions noisy as genes and FRs may exhibit considerable variations across studies. Such an approach also relies implicitly on prior information, hence may have limited use in discovering novel FRs. Recent studies have provided compelling evidence of non-canonical signalling mechanism and cross-talk between pathways $[3,4]$ that demand inferring network structure from the given data as opposed to direct inference from documented/ curated pathways.

Bayesian structure learning (BSL) techniques [5] have been used successfully to infer interactions between a given set of genes in the form of graphs. The inherent probabilistic nature of gene expression and access to high-throughput assays that facilitate simultaneous measurement of transcriptional, translational and posttranslational activities [4] are some of the reasons for their wide-spread use. Gene expression data with interventions [4] have also been recently shown to improve the conclusions drawn using Bayesian network modelling [4]. Probabilistic mechanisms underlying gene expression can be attributed to inherent noisiness and heterogeneity within/between cell population(s) [6]. High-throughput assays such as microarrays $[7,8]$ and clonal gene expression profiling [9] in conjunction with BSL [10] had been used successfully in the past to capture functional relationships at the transcriptional level. More recently, high-throughput flow cytometry data from single-cells with perturbations in conjunction with BSL were used to obtain system-level understanding at translational and post-translational data [4].

Several open-source packages are available for BSL and can be used to model gene networks [11-13]. However, these packages expect the user to be familiar with the programming environment and syntax. NATbox, Fig. 1, aims to overcome some of these obstacles by providing a menu-driven GUI for modelling and analysis of gene expression networks. It invokes functions from existing R-packages to accomplish this [14]. Reusing existing functionalities is strongly encouraged in an open-source environment and can minimize redundancy while improving transparency, reproducibility and sustainability. The target audience of NATbox are interdisciplinary researchers and biologists who wish to explore the tools of systems biology and network modelling for investigating signalling mechanisms. NATbox does not require the user to be familiar with R-programming or the algorithmic intricacies. Suitable parameter recommendations along with popular default parameter choices are provided as required to the user. As with any R-package, NATbox is open-source, hence lends itself to be customized by users with expertise in R. A detailed Wiki help page accompanies the toolbox and provides a systematic overview of the toolbox functions along with examples AT http://bioinformatics.ualr.edu/natboxWiki/ index.php/Main_Page.

\section{Results}

Prior to a detailed description of NATbox functionalities, we briefly review those of a closely related package BNArray [13] which was designed to provide a higherlevel abstraction of R-routines from existing packages for 


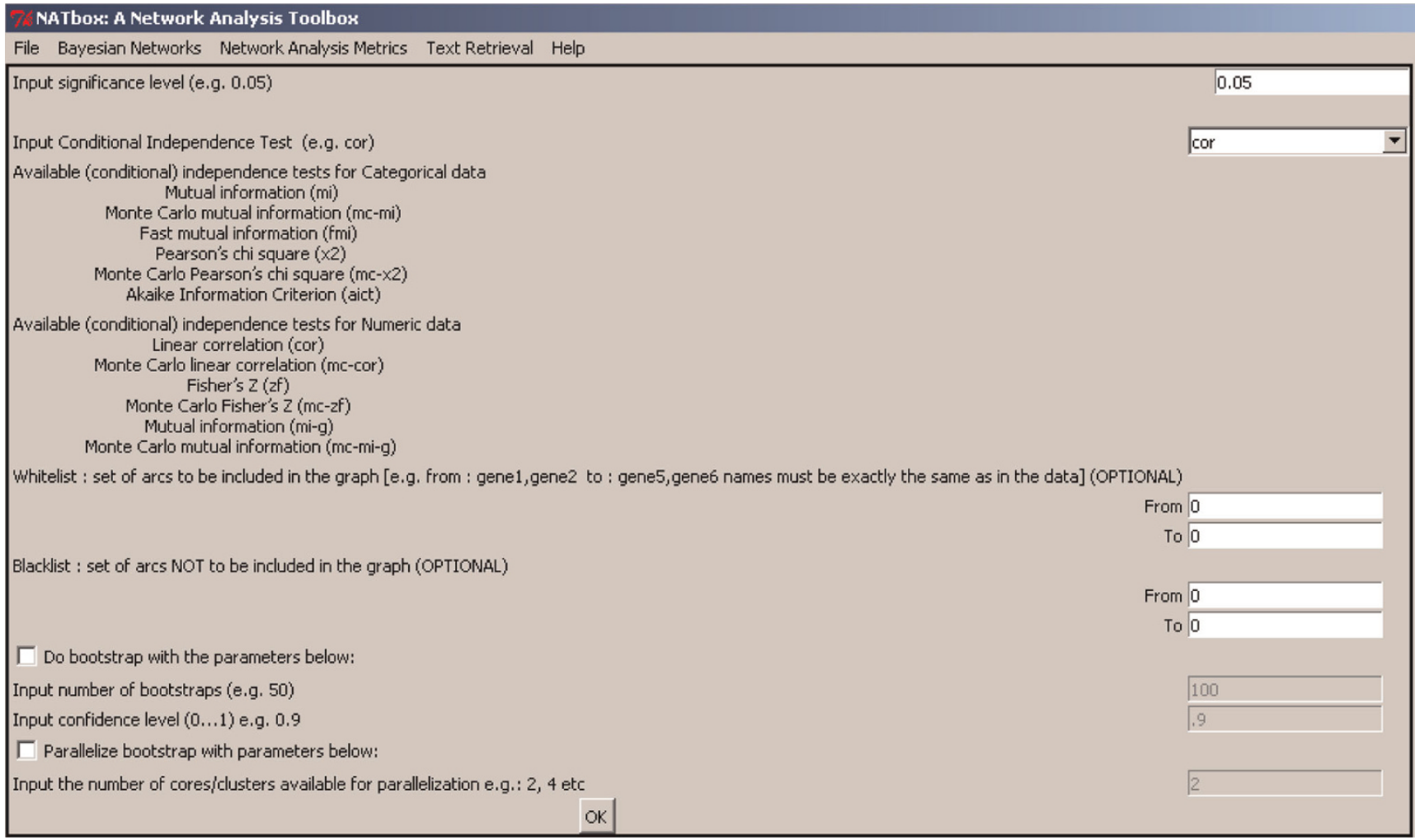

Figure I

Network Analysis Toolbox (NATbox). A screen shot of the Network Analysis Toolbox (NATbox) showing the main menu and the interface for Bayesian structure learning (BSL) with parameter recommendations and default values.

gene network modelling. BNArray is a command line driven package where the user is expected to be familiar with the $\mathrm{R}$ environment [14] and syntax. It consists of four main modules $(i)$ determine missing values in a given data using the functions LLSimpute, also implemented in the R-package pcamethods [15] (ii) Learn acyclic network structure using BSL routines from the R-package deal [16]. Subsequently display the graph by invoking functions from the R-package dynamicGraph [17] (iii) identify what the author term as $1^{\text {st }}$ order network structure using a resampling procedure and (iv) reconstruct coherent sub-regulatory networks using an extended version of the algorithm CODENSE [18]. In the following discussion, we describe the features of NATbox as well as compare those implemented in BNArray in a systematic manner.

The comparisons are also enclosed under Table 1 for a quick review.

i. GUI: NATbox provides a convenient menu-driven graphical user interface (GUI) developed using Tcl/Tk for modelling and analysis of gene expression networks. This has to be contrasted with BNArray [13], which is a command line driven package and demands the user to be familiar with the R-environment and syntax.

ii. Input/output: The input data in NATbox is assumed to be in tab-delimited text format, similar to that of BNArray. In NATbox, the rows of the input file represent independent experiments whereas the columns represent the names of the genes. However, in BNArray the rows represent the genes of interest and the columns represent their expression across experiments. Unlike NATbox, BNArray does not specify whether the experiments need to be independent or dependent.

iii. Missing values: Gene expression profiles often have missing values. For instance, in microarray data such missing values are common and may be attributed to experimental artefact, improper hybridization and nonspecific binding of the probes. It is prudent to use suitable statistical techniques to accommodate such data sets rather than discard them. NATbox provides an option to determine missing values using nearestneighbour averaging approach (menu: File), impute.knn (R-package impute) which has been found to perform well for high-dimensional data sets [19]. BNArray also 
Table I: Comparison of main features in NATbox and BNArray

\section{NATbox}

Provides a Graphical User Interface (GUI)

Supported across operating systems: Windows and Linux.

Parameter recommendations are provided for the various algorithms with default values automatically inserted in GUI.

Input file is tab-delimited where columns represent the genes of interest and rows represent the experiments. The rows are assumed to be independent of one another.

Imputation of missing values is accomplished by k-nearest neighbour approach impute.knn from the R-package impute

Bayesian Structure Learning (BSL) algorithms are invoked from the R-Package bnlearn.

BSL algorithms ideally suited for continuous as well as discrete data sets characteristic of gene expression profiles and their quantized/coarse-grained counterparts.

BSL algorithms from the package bnlearn include constraint-based (GS, IAMB, Fast-IAMB, IIAMB, MMPC) as well as search and score techniques

(Hill-Climbing) are implemented.

BSL techniques implemented from bnlearn provide choice of several conditional independence tests and scoring criteria for continuous and discrete data sets under constraint-based and search and score techniques respectively.

Conditional independence tests for constraint-based: mutual information, mutual information for Gaussian distributed data, fast mutual information, Pearson $X^{2}$, Akaike information criterion, linear correlation and Fisher's Z.

Scoring Criteria for search and score: multinomial likelihood, multinomial log-

likelihood, Akaike information criterion, Bayesian information criterion, Bayesian

Dirichlet score and Gaussian posterior density).

Provides options to incorporate structural priors during BSL by whitelisting (include) and blacklisting (exclude) edges.

Confidence of an edge is determined by bootstrapping. Uses R-package RGraphviz for visualization, which is designed to handle the layout of large graphs.

Provides options to highlight edges whose confidence is greater than user specified threshold.

Parallelization of the bootstrap routines is accomplished by invoking functions from the R-package SNOW.

Topological properties of the results of BSL are investigated using centrality measures (degree, betweenness and closeness) from the R-package igraph. Provides motif finder from the package igraph for identifying motifs from the results of BSL. Results from igraph can also be loaded into Cytoscape fro detailed visualization.

Provides a text retrieval interface to retrieve published literature to retrieve functional relationships of interest. This is useful justifying the choice structural priors in BSL.

\section{BNArray}

Command-line driven with no graphical user interface (GUI). Supported across operating systems: Windows and Linux. No parameter recommendations and default values are provided for the algorithms. Input file is tab-delimited where rows represent the genes of interest and columns their measurements across experiments. Unlike NATbox, no specifications are provided as to whether the experiments need to be correlated or uncorrelated. Imputation of missing values is accomplished by local least square estimation LLSImpute also implemented in the R-package pcamethods.

Bayesian Structure Learning (BSL) algorithms are invoked from the R-Package deal.

BSL algorithm is ideally suited for mixed data type consisting of continuous as well as discrete values. Although, the authors of BNArray use it for analyzing temporally correlated gene expression data.

No options are provided for multiple BSL algorithms.

No options are provided for multiple BSL algorithms.

No options are provided for incorporating structural priors.

Confidence of an edge is determined by bootstrapping. Uses R-package dynamicGraph for visualization, which may require manual tuning of the node layout for large graphs.

No options are provided to highlight edges whose confidence is greater than user specified threshold.

No options are provided for parallelization.

Does not provide any centrality measures.

Provides a modified version of the algorithm CODENSE for constructing coherent sub-networks from the results of BSL.

No interface for text retrieval is provided.

provides a neighbourhood-based imputation technique, namely the least local squares (LLSImpute) [15] algorithm for determining missing values. A version of LLSImpute algorithm is also available through the (R-package pcamethods).

iv. Functional relationships: NATbox provides the option to model functional relationships using a battery of Bayesian structure learning techniques from the R-package (bnlearn) [20] (menu: Bayesian Networks). It is important to note that they model the network structure solely from the joint probability distribution in the absence of explicit temporal information. The tab- delimited input data should be in the form of a matrix where the columns represents the number of genes, and rows the number of repeated (independent) experiments. Each element in the matrix represents the expression value of that gene in a given experiment.

Constraint-based techniques in NATbox that model the network based on the results of conditional independence tests include:

- Grow-Shrink Algorithm (GS) [21]: GS is based on the Grow-Shrink Markov blanket, the first (and simplest) Markov blanket detection algorithm. 
- Incremental Association Markov Blanket Algorithm (IAMB) [22]: IAMB is based on the Markov blanket detection algorithm. It uses a two-phase selection scheme. A forward selection followed by an attempt to remove false positives which scales well up to thousands of variables.

- Fast Incremental Association Markov Blanket Algorithm (Fast-IAMB) [23]: Fast-IAMB is a variant of IAMB which uses speculative stepwise forward selection to reduce the number of conditional independence tests.

- Interleaved Incremental Association Markov Blanket Algorithm (Inter-IAMB) [22]: Inter-IAMB is another variant of IAMB that uses forward stepwise selection to avoid false positives in the Markov blanket detection phase. Even though it often requires more conditional tests than the other IAMB variants, it's still able to scale up to thousands of variables while maintaining its robustness against false positives.

- Max-Min Parents Children Algorithm (MMPC) [24]: MMPC is a forward selection technique for neighbourhood detection based on the maximization of the minimum association measure. It learns the underlying structure of the Bayesian.

Several choices of conditional independence tests for categorical/discrete (mutual information, mutual information for Gaussian distributed data, fast mutual information, Pearson's $\chi^{2}$, Akaike information criterion) as well as numerical/continuous distributions (linear correlation, Fisher's Z) are provided. NATbox also provides an interface to the search and score algorithm (HC, Hillclimbing) from bnlearn. HC searches the model space and retrieves the best model using a scoring criterion which is also provided. Several choices of scoring criterion are provided for categorical/discrete (multinomial likelihood, multinomial log-likelihood, Akaike information criterion, Bayesian information criterion, Bayesian Dirichlet score, K2 score) and numerical/continuous distributions (Gaussian posterior density). Each BSL technique works under implicit assumptions and may result in spurious conclusions when these assumptions are violated. NATbox provides a battery of BSL techniques to alleviate such concerns. For instance, constraint-based techniques can be affected by sample sizes and are sensitive to initial results of the conditional independence tests. Search and score algorithms can result in local optima, hence may benefit from multiple random restarts unlike constraintbased approaches.

On the other hand, BNArray [13] uses a greedy search implementation of the BSL technique implemented in deal to learn the network structure. It is important to note that algorithm implemented in deal is ideally suited for handling mixed data types consisting of continuous as well as discrete data types. Although the authors [13] of BNArray have demonstrated the performance of their approach on temporal gene expression profile, it is unclear as to whether deal algorithm accommodates such temporal explicit information during structure learning. This is in contrast with NATbox which implements algorithms from bnlearn for learning the network structure from homogenous data types characteristic of gene expression profiles, i.e. either continuous or discrete data sets.

$v$. Bootstrap parallelization: Bootstrap techniques are commonly used for confidence estimation from a given empirical sample. Confidence [7] of an edge is determined by bootstrapping the given empirical data with replacement. Edges whose confidence is greater than a userdefined threshold $(0<\theta<1)$ are deemed robust. Bootstrap procedures can be computationally demanding for highdimensional data sets. Search and score techniques such as hill-climbing implicitly require several random restarts during the confidence estimation. NATbox provides an option to parallelize bootstrapping across multiprocessor or multi-core processor by invoking the appropriate routines from the R-package SNOW (Simple Network Of Workstations) [25]. Such parallelization fall under embarrassingly parallel problems. However, they can significantly reduce the overall time complexity. Parallelization of the BSL routines themselves was not found to yield any significant improvement with increasing number of processors. We believe this may be due to inherent overhead in the master-worker configuration in SNOW. This is still under investigation. The performance for the various structure learning algorithms with increasing number of bootstraps and processors on BSL of the protein expression data [4] is shown in Fig. 2. A marked decrease in computational time is observed with increasing number of processors. The computational time with 1 processor is almost 5 folds of that obtained by 8 processors across the three algorithms (GS, IAMB and MMPC) and 1000 bootstrap simulations.

The results of the bootstrap are written onto a tabdelimited file. An option is also provided for highlighting the robust FRs $(\theta>0.8)$ on the acyclic graph learned from the given data, Fig. 3. BNArray also implements bootstrap routines to determine what the author call as first-order network structures. Unlike NATbox, BNArray does not provide any options for bootstrap parallelization. BNArray uses routines from the package dynamicGraph [17] for visualization of the resulting acyclic network.

In addition, NATbox GUI also provides options for incorporating (directional) structural priors in BSL by 


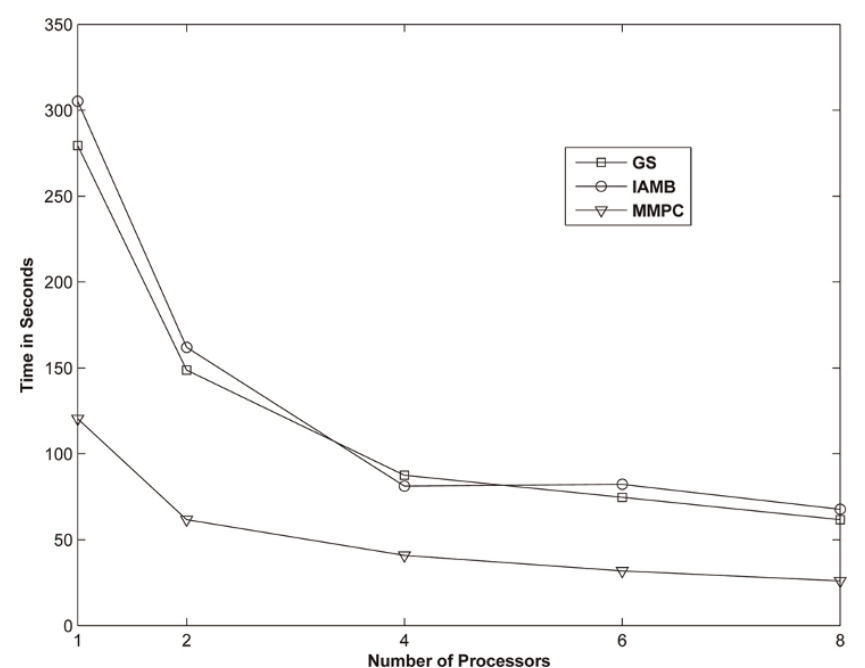

Figure 2

Performance of BSL with bootstrap parallelization. The performance of three BSL techniques (GS, IAMB and MMPC) with increasing number of processors ( $n p=1,2,4,6$ and 8 ) and 1000 bootstrap simulations, across an 8-processor Linux machine for the data [4]. Each of these algorithms exhibits ( $\sim 5$ fold) decrease in computational time at $(n p=8)$ compared to $(n p=I)$.
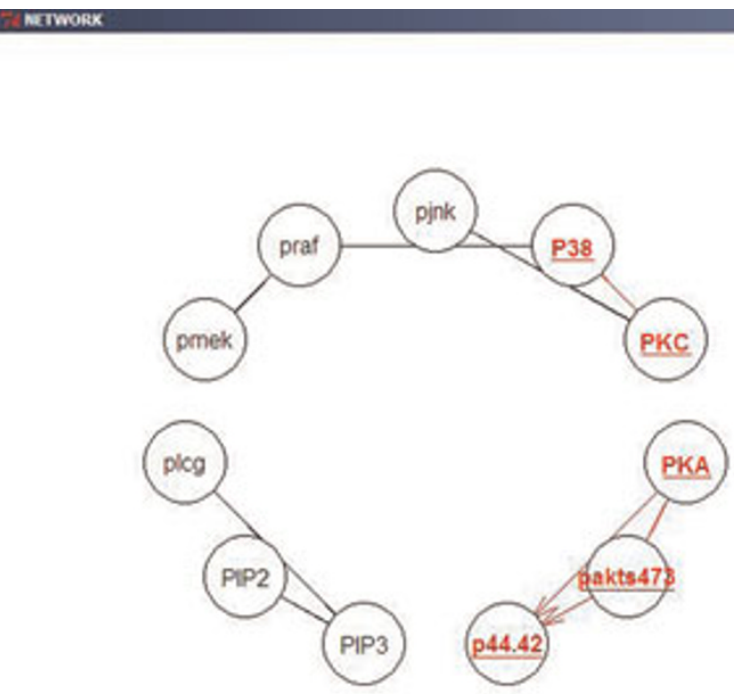

Coor to clooourd

Figure 3

Highlight Robust FRs. The network structure learned using the Grow-Shrink (GS) algorithm from the data [4]. Robust FRs are deemed as those whose confidence is greater than the user-defined threshold $(\theta>0.8)$, highlighted in red. whitelisting (include) and blacklisting (exclude) FRs. Such priors impose constraints on the network structure which implicitly rely on prior knowledge and needs to be chosen prudently in order to avoid bias during learning. However, a proper choice can alleviate uncertainty and improve accuracy of the conclusions. A text retrieval interface is provided for identifying structural priors and can be useful for investigating well-established signalling mechanisms.

vi. Network analysis metrics: BSL techniques are useful in inferring the cause-effect relationships and network structure from the gene expression profiles. However, they provide no insight into the network's topological properties. NATbox incorporates social network analysis metrics and motif finder from the package (igraph) [26] under (menu: Network Analysis Metrics) for investigating the topological properties of the networks generated using BSL techniques. Such metrics can be especially useful in investigating large networks. The input is assumed to be a binary adjacency matrix of the network constructed using BSL with ones and zeros representing the presence/absence of an edge respectively. Since BSL results in directed acyclic graphs, the corresponding binary adjacency matrix need not be symmetric. The centrality measures (degree centrality, closeness centrality, betweenness centrality, alpha centrality) along with their respective parameter options are provided for the user. A detailed discussion of these centrality measures are deferred to $[26,27]$. Degree centrality is one of the commonly used measures of centrality and broadly classified into out-degree (edges directed outward from a node) and in-degree (edges directed into a node). The distribution of the degree centrality has been widely studied and provides crucial insight into the topology of a network. Recent studies have elucidated the prevalence of power-law degree distribution in biological networks [28]. Such power-law distributions may be accompanied by interesting properties such as the presence of hubs (critical genes) and scale-free phenomena unlike exponential degree distributions. Justification of power-law degree distributions can be challenging especially when the information about the tail of the distribution is insufficient [29]. Betweenness centrality is useful in identifying genes that act as important mediators between other genes, although they themselves might not be densely connected to all the packages. Genes with high betweenness centrality may play an important role in bridging the gap between densely connected subgroups. On the other hand, closeness centrality is useful in identifying genes that are directly or indirectly dependent on other genes. The menu also provides an option to determine the diameter and identify recurrent structures or motifs of sizes 3 and 4 in the given network by invoking the respective functions from igraph [26]. 


\title{
pmek-P38
}

\author{
$\underline{16864584}$
}

\section{PIP2-PIP3}

\section{$\underline{19340548}$}

$\underline{19295169}$

$\underline{19133120}$

$\underline{19129674}$

$\underline{18794878}$

$\underline{18695396}$

$\underline{18063934}$

$\underline{18004876}$

\section{$\underline{17366487}$}

\section{1: 1 Biol Chem. $2006 \operatorname{Sep} 29 ; 281(39): 29085-95$. Epub 2006 Jul 24.}

Induction of Krox-24 by endogenous cannabinoid type 1 receptors in Neuro2A cells is mediated by MAPK pathway and is suppressed by the phosphatidylinositol 3-kinase pathway.

Graham ES, Ball N, Scotter EL, Narayan P, Draqunow M, Glass M.

Department of Pharmacology, University of Auckland, Private Bag 92019, Auckland, New Zealand.

Neuro2a cells endogenously express cannabinoid type 1 (CB1) receptors. CB1 stimulation with HU2 and induced the transcription factor Krox-24. A functional MEK-ERK pathway is an important require CB1-mediated Krox-24 induction as blockade of MEK signaling by UO126 reduces both basal and CE activation of Krox-24. CB1 receptor stimulation did not activate either JNK or p38 MAPK pathways o pro-proliferation phosphatidylinositol 3-kinase (PI3K)-Akt pathway. However, serum removal or blo signaling by LY294002 transiently stimulated basal Krox-24 expression and increased CB1-mediate Krox-24. This was consistent with a transient increase in PMEK, DERK, and PCREB levels following P These data demonstrate that CB1-mediated activation of the Krox-24 transcription factor is negativ through the PI3K-Akt pathway and reveals several points of signaling cross-talk between these tw pathways.

\section{Figure 4}

Text retrieval interface for structural priors. Results of text retrieval interface (html file) revealing prior literature (PubMed identifiers) on one of the robust FRs (PIP2-PIP3) identified by BSL of the expression data [4]. These results in turn can be used to impose structural priors, hence refine BSL.

Motifs are recurrent atomic structures with frequency distribution different from that of random network. Recent studies have identified certain specific motifs that persist across distinct real world networks [30]. The igraph package incorporated into NATBox also provides option to save the acyclic network in formats compatible with Cytoscape [31] for detailed visualization.

vii. Retrieving FRs from literature: Finally, NATbox provides options for retrieving FRs of interest using NCBI ESearch (menu: Text Retrieval). The user has the option to input the (a) pairs of gene names (co-occurrence) of interest through the GUI or (b) upload a two-column matrix of FRs of interest or those identified as robust by the bootstrap procedure. For well-documented studies, an integrated approach that incorporates the results from the Text Retrieval (structural prior) in justifying the choice of whitelisted (include) and/or blacklisted (edges) in BSL. The Text Retrieval results are in html format, with a list of PUBMED identifiers hyper-linking to the respective abstracts/articles in PUBMED. The results of text retrieval on gene expression data from [4] is shown in Fig. 4.

\section{Conclusion}

Modelling and analysis of gene expression networks is an area of active research. Several tools have been proposed in the literature for the same. Recently, Bayesian structure learning (BSL) techniques in conjunction with high throughput assays were used successfully to capture functional relationships. Existing packages may demand the user to have considerable programming expertise. NATbox provides a convenient menu-driven GUI along with appropriate parameter recommendations including default parameter choices for modelling and analysis of gene expression networks. It incorporates diverse functionalities from existing R-packages. This in turn encourages transparency and reproducibility, characteristic of open-source environment. NATbox can also be used as a teaching and demonstration tool for graduate courses in systems biology. Immediate future 
enhancements to the toolbox include (i) expand the choice of structure learning algorithms including dynamic bayesian networks (ii) improve statistical inference of the network features (ii) parallelization of the implemented routines across multi-core and multiprocessor machines of BSL functions as well as bootstrapping (iii) provide a web-interface so as to obviate the need for local installation of the toolbox (iv) enhance text retrieval so as to accommodate advanced text mining approaches.

\section{Competing interests}

The authors declare that they have no competing interests.

\section{Authors' contributions}

SSC and MAB implemented the toolbox under RN's guidance. MS developed the bnlearn package and was involved in trouble shooting the Bayesian structure learning routines. $\mathrm{RN}$ wrote the manuscript.

\section{Acknowledgements}

RN acknowledges funding from R03L008853 and \#P20-RRI6460 from IDeA Network of Biomedical Research Excellence (INBRE) Program of the National Center for Research Resources (NCRR/NIH).

This article has been published as part of BMC Bioinformatics Volume 10 Supplement II, 2009: Proceedings of the Sixth Annual MCBIOS Conference. Transformational Bioinformatics: Delivering Value from Genomes. The full contents of the supplement are available online at http://www.biomedcentral.com/I47I-2I05/I0? issue=SII.

\section{References}

I. Subramanian A, et al: Gene set enrichment analysis: a knowledge-based approach for interpreting genome-wide expression profiles. Proc Nat Acad Sci USA 2005, I 22(43): I5545-50.

2. Carbon S, et al: AmiGO: online access to ontology and annotation data. Bioinformatics 2009, 25(2):288-9.

3. Zhou , et al: Modulation of morphogenesis by noncanonical Wnt signaling requires ATF/CREB family-mediated transcriptional activation of TGF $\beta 2$. Nature Genetics 2007, 39: 1225-1234.

4. Sachs K, et al: Causal Protein-Signaling Networks Derived from Multiparameter Single-Cell Data. Science 2005, 308:523-529.

5. Pearl J: Causality: Models, Reasoning, and Inference. Cambridge University Press; 2000.

6. McAdams $\mathrm{H}$ and $\mathrm{H}$ Arkin AP: Genetic Regulation at the Nanomolar Scale: It's a Noisy Business! Trends in Genetics 1999, I 5(2):65-69.

7. Friedman N, et al: Data Analysis with Bayesian Networks: A Bootstrap Approach. Proc Fifteenth Conf on Uncertainty in Artificial Intelligence (UAl) 1999, 196-205.

8. Friedman N, et al: Using Bayesian Network to Analyze Expression Data. J Computational Biology 2000, 7:60I-620.

9. Madras N, et al: Modeling Stem Cell Development by Retrospective Analysis of Gene Expression Profiles in Single Progenitor-Derived Colonies. Stem Cells 2002, 20:230-240.

10. Nagarajan R, et al: Modeling genetic networks from clonal analysis. J Theor Biol 2004, 230(3):359-73.

II. Hartemink A: Bayesian Network Inference with Java Objects (BANJO). http://www.cs.duke.edu/ amink/software/.

12. Murphy K: Bayes NET Toolbox for MATLAB. http://www.cs. ubc.ca/ murphyk/Software/BNT/bnt.html.
13. Chen $X$, et al: BNArray: an $\mathbf{R}$ package for constructing gene regulatory net-works from microarray data by using Bayesian network. Bioinformatics 2006, 22(23):2952-4.

14. R Development Core Team: R: A language and environment for statistical computing. R Foundation for Statistical Computing; 2008

15. $\mathrm{Kim} \mathrm{H}$, et al: Missing value estimation for DNA microarray gene expression data: local least squares imputation. Bioinformatics 2005, 21(2): | 87-198.

16. Bøttcher SG and Dethlefsen C: Deal: a package for learning Bayesian Networks. J Stat Software 2003, 8: $1-40$.

17. Badsberg JH: DynamicGraph, CRAN package, 0.2.2.5 2009

18. $\mathrm{Hu} \mathrm{H}$, et al: Mining coherent dense subgraphs across massive biological networks for functional discovery. Bioinformatics 2005, 2 I (Suppl):i2 I3-i22 I.

19. Hastie T, Tibshirani R, Sherlock G, Eisen M, Brown P and Botstein D: Imputing Missing Data for Gene Expression Arrays Stanford University Statistics Department Technical Report; 1999.

20. Scutari M: bnlearn: Bayesian network structure learning, Version I.3, CRAN package 2008.

21. Margaritis D: Learning Bayesian Network Model Structure from Data. Ph.D. Thesis, School of Computer Science, Carnegie-Mellon University, Pittsburgh, PA, 2003. Technical Report CMU-CS-03-153.

22. Tsamardinos I, et al: Algorithms for Large Scale Markov Blanket Discovery. Proceedings of the Sixteenth International Florida Artificial Intelligence Research Society Conference 2003, 376-38I.

23. Yaramakala $S$ and Margaritis D: Speculative Markov Blanket Discovery for Optimal Feature Selection. Proceedings of the Fifth IEEE International Conference on Data Mining 2005, 809-8I2.

24. Tsamardinos I, et al: The Max-Min Hill-Climbing Bayesian Network Structure Learning Algorithm. Machine Learning 2006, 65(I):31-78.

25. Tierney, et al: snow: Simple Network of Workstations, CRAN package $0.3-32008$.

26. Csardi G: igraph: Routines for simple graphs, network analysis Version 0.5. I. CRAN package 2008.

27. Wasserman S and Faust K: Social Network Analysis Cambridge University Press; 2007.

28. Barabási A-L, et al: Network biology: understanding the cell's functional organization. Nature Reviews Genetics 2004, 5: $|0|-|| 3$.

29. Goldstein ML, et al: Problems with fitting to the power-law distribution. The European Physical Journal 2004, B 4 I:255-258.

30. Milo R, et al: Network Motifs: Simple Building Blocks of Complex Networks. Science 2002, 298:824-827.

31. Shannon P, et al: Cytoscape: a software environment for integrated models of biomolecular interaction networks. Genome Research 2003, I 3(I I):2498-504.

32. Margaritis D: Learning Bayesian Network Model Structure from Data. Ph.D. Thesis, School of Computer Science, Carnegie-Mellon University, Pittsburgh, PA 2003, Available as Technical Report CMUCS-03- 153.

33. Tsamardinos I, Aliferis CF and Statnikov A: “Algorithms for Large Scale Markov Blanket Discovery". "Proceedings of the Sixteenth International Florida Artificial Intelligence Research Society Conference" AAAI Press; 2003, 376-38I.

34. Yaramakala $S$ and Margaritis D: "Speculative Markov Blanket Discovery for Optimal Feature Selection". "ICDM '05 Proceedings of the Fifth IEEE International Conference on Data Mining" IEEE Computer Society, Washington, DC, USA; 2005, 809-8I2.

35. Tsamardinos I, Brown LE and Aliferis CF: "The Max-Min HillClimbing Bayesian Network Structure Learning Algorithm". Machine Learning 2006, 65(1):31-78. 